\title{
Haemorrhagic stroke during pregnancy may be successful - a case study of two cases
}

\begin{abstract}
Stroke is a disease, which is characteristic in older and middle-aged people; however, it can also occur in younger people. Pregnancy and puerperium are its important risk factors. The most important risk factor of stroke in pregnant women is preeclampsia and eclampsia. These conditions may develop into the HELLP syndrome, which is characterized by a simultaneous occurrence of hemolysis, elevated liver enzymes, and low platelet count. The authors present two pregnant women who developed eclampsia with HELLP syndrome and in who haemorrhagic stroke occured. The process of treatment in described patients was supervised by: a neurologist, a gynecologist, an anesthesiologist and a hematologist. The cesarean section was expertly performed within a very short time after the arrival to hospital. After the treatment, in both the patients, blood parameters normalized. In both the cases, prompt and multidirectional action was taken. We would like to emphasize the fact that pregnant patients with eclampsia and HELLP syndrome should be directed to multispecialist center as soon as possible, as they need a comprehensive treatment. The authors draw attention to the need for rapid diagnosis and immediate implementation of a multi-specialty treatment in these patients, in which cesarean section act pivotal role.
\end{abstract}

Special Issue - 2015

\author{
Wiszniewska Malgorzata,' Izabela \\ Stachowiak, ${ }^{2}$ Beata Nowak, ${ }^{3}$ Pawel \\ Wiszniewski ${ }^{4}$ \\ 'Neurological Department, Specialist Hospital Pia, Poland \\ ${ }^{2}$ Gynaecological Department, Specialist Hospital Pia, Poland \\ ${ }^{3}$ Emergency Department, Specialist Hospital Pia, Poland \\ ${ }^{4}$ Internal and Haematology, Department Specialist Hospital Pia, \\ Poland
}

Correspondence: Wiszniewska Malgorzata, Neurological Department, Specialist Hospital Pila, Poland, Emailmpwisz@gmail.com

Received: February 19,2015 | Published: June 08, 2015

Keywords: stroke, intracerebral hemorrhage, eclampsia, pregnancy, risk factors, HELLP syndrome

Abbreviations: aPTT, activated partial thromboplastin time; $\mathrm{PT}$, prothrombin time; MR, magnetic resonance imaging; mRs, modified rankin scale; DIC, disseminated intravascular coagulation

\section{Introduction}

Stroke in pregnancy and puerperium appears in $10-34 / 100,000$ parturient woman and it is a major cause of morbidity and mortality at them. ${ }^{1}$ The most important risk factor for pregnant women is preeclampsia and eclampsia. ${ }^{1-3}$ Preeclampsia is a condition where blood pressure amounts $\geq 140 / 90 \mathrm{mmHg}$ measured twice every 4 hours over 20 weeks of pregnancy without prior hypertension, together with proteinuria $>300 \mathrm{mg} / 24 \mathrm{~h}$. Eclampsia refers to the occur of one or more generalizated convulsion and/or coma in the setting of preeclampsia and in the absence of other neurologic condition. It can appear as a separate disease, without preeclampsia before. The incidence of eclampsia has been stable at $1.5-10$ cases per 10,000 deliveries in developed countries. ${ }^{4,5}$ These conditions may develop to the HELLP syndrome, which is characterized by a simultaneously prevalence of hemolysis, elevated liver enzymes, and low platelet count. ${ }^{6-8}$ In both preeclamsia /eclampsia as well as in HELLP the hypoxia of placenta and trophoblast is developing, and then inflammatory cytokinins are produced and develops dysfunction of maternal endothelium. In the further are synthesized vasoconstrictors and unfurl systemic inflammatory response. ${ }^{7,8}$ Autoregulation and perfusion in the brain is distorted. ${ }^{6,7}$ When perfusion is increased is developing hemorrhagic stroke, when reduced creates ischaemic lesions. Besides in HELLP can arises DIC and secondary haemorrhagic diathesis what also contributes to cerebral haemorrhage. ${ }^{9}$ HELLP syndrome occurs in $4-12 \%$ of pregnant woman with severe preeclampsia. In some cases HELLP syndrome symptoms may be a separate entity. Morbidity and mortality rated associated with the syndrome is over 25 percent.

It is estimated that in $30 \%$ of gravids with eclampsia/preeclampsia unrolls stroke. , $, 2,4,10,11$ In eclampsia and preeclampsia especially with HELLP syndrome most often coming to the development of haemorrhagic stroke. When clinically state suggests stroke at pregnant women with preeclampsia/eclampsia it is necessary immediately implement hypotensive treatment, intravenous magnesium sulphate infusions, and a pregnancy resolved by cesarean section. ${ }^{10}$ Careful observation and rapid management can save mother and the baby with severe complication of HELLP syndrome and stroke. It is also needful a good co-operation between many experts in such cases.

The authors present two pregnant women at who has evolved eclampsia with HELLP syndrome and occurred haemorrhagic stroke. The authors draw attention to the need for rapid diagnosis and immediate implementation multi-specialty treatment in these patients.

\section{Case reports}

\section{Case one}

A 38-year-old woman (gravididas 7, 32 week) has been imported by ambulance from home to the hospital because of the first in the life two tonic-clonic epileptic seizures which occurred suddenly afternoon. The increase of blood pressure $160 / 115 \mathrm{mmH}$ was accompanied by the seizures. Her past medical history had been unremarkable: she had the correct blood pressure, and gynecologist assessed her status as a good one. During the ambulance transport $\mathrm{MgSO} 4 \mathrm{lg}$ was administrated intravenously. On admission in emergency department it appeared the second generalizing epileptic. The general state was serious, her blood pressure was 160/115mmHg. She was in cerebral coma (Coma Glassgow Scale (CGS)-5), and presented right hemiplegy. Based on the clinical presentation and laboratory tests it was recognized eclampsia with HELLP syndrome and stroke. In blood investigations at the admission: $\mathrm{Hb}-13,5 \mathrm{~g} / \mathrm{dl}$ (n.11,2-15,7), E-3,41M/uL (n. 4,005,00), L-16,32K/uL (n.3,9-10,04), platelets-103K/uL (n. 140-370), coagulogram: activated partial thromboplastin time (aPTT)-31,4s (n.24,0-36,9), prothrombin time (PT)-9,8s (n. 8,9-13,1), fibrinogen- 
279mg/dl (n.180-390), D-Dimer $16864 \mathrm{EU} / \mathrm{ml}(\mathrm{n} .<500)$, the liver tests were invalid: ALAT-1032IU/1 $(\mathrm{n} .<33)$, ASPAT-1257 IU/1 (n. $<32)$. Protein in urine was present $(0.75 \mathrm{~g} / \mathrm{l})$. She was immediately transported to operating room and one hour and 15 minutes after patient's arrival she underwent a cesarean section because of the presence of non reassuring fetal status and hypertension. A live female baby was delivered. After operation the patient was treated in intensive care department- 8 days following neurological -8 days. On the third day decreased: platelets to values $47 \mathrm{~K} / \mathrm{ul}, \mathrm{Hb}$ to $9,6 \mathrm{~g} / \mathrm{dl}$. She received infusions of $\mathrm{MgSO}_{4}$, concentrate of red blood cells and platelets. Due to convulsion besides $\mathrm{MgSO}_{4}$ it was used clonazepam following valproic acid. Her blood pressure was reduced to within the normal range by using: indapamidum, amlodipinum, ramiprilum, methylodopum. CT image obtained after cesarean section depicted signs of subarachnoid haemorrhage, haemorrhagic focus in left occipital lobe, and hypodensive area in the bilateral occipital lobes (Figure 1a). Magnetic resonance imaging (MR) demonstrated regular intracerebral arteries and haemorrhagic focus in left occipital lobe (Figure 1b). At 16 days postpartum, the mother and her baby were discharged without any neurological abnormalities. 9 months later thy both are in very good condition and the mother doesn't require any drug.
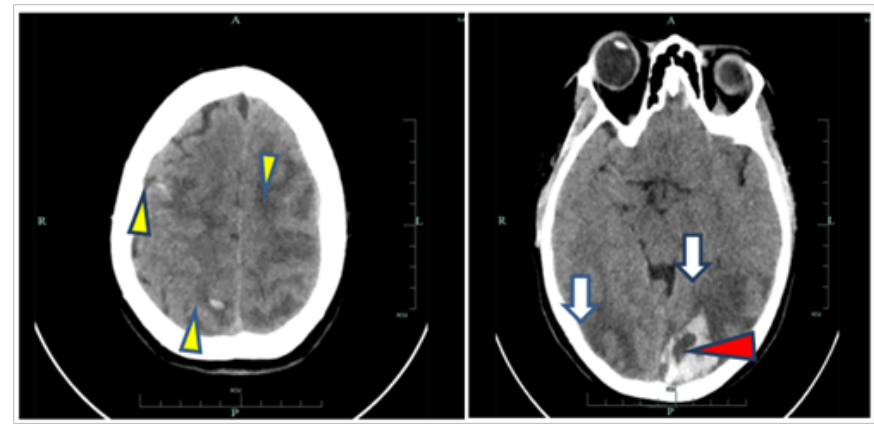

Figure Ia CT image after cesarean section: yellow triangle - signs of subarachnoid haemorrhage, red triangle - haemorrhagic focus in left occipital lobe, white arrow - hypotensive area in the bilateral occipital lobes.

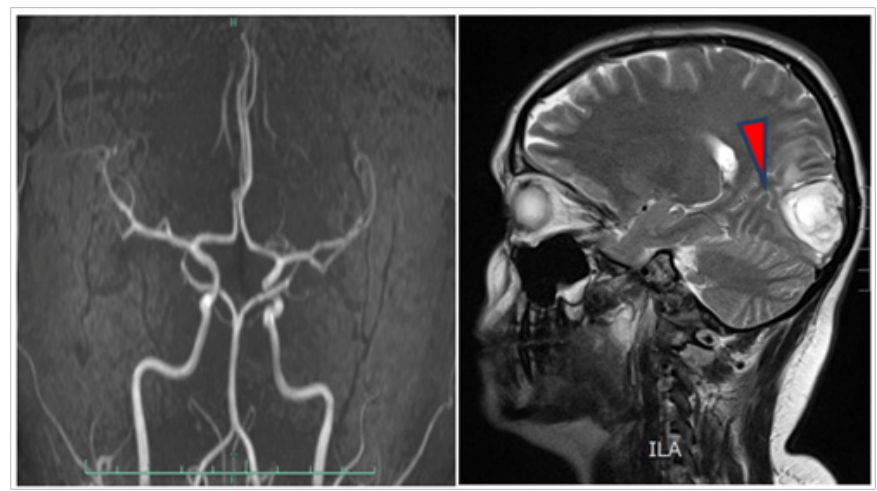

Figure Ib Magnetic resonance imaging (MR) at 16 days postpartum: haemorrhagic focus in left occipital lobe, regular intracerebral arteries.

\section{Case second}

A 23-year-old woman (gravididas1, 37 week) was admitted to Neurological department from Gynecology due to right hemiplegy with aphasia and with accompanying hypertension which prior didn't appear. At the admission the general state was serious; she was comatose, blood pressure 200/110mmHg, CGS -5, right hemiplegy with eyeball movement disturbances. It was recognized eclampsia with HELLP syndrome and stroke. In blood investigations during admission: $\mathrm{Hb}-6,6 \mathrm{~g} / \mathrm{dl}, \mathrm{E}-2,16 \mathrm{M} / \mathrm{ul}, \mathrm{L}-7,9 \mathrm{~K} / \mathrm{ul}$, platelets- $44 \mathrm{~K} /$ ul, coagulogram: aPTT-32,1 s, PT-10,0 s, fibrinogen-310mg/dl, D-Dimer-16728 ngFEU/m, ALAT-153IU/1, AsPAT-414 IU/1. 35 minutes since the admission to hospital she underwented a cesarean section. The patient was treated in intensive care department 4 days and then on neurology 19 days. She received infusions of $\mathrm{MgSO}_{4}$, concentrate of red blood cells and platelets, bisoprolol. Due to convulsion besides $\mathrm{MgSO}_{4}$ it was used clonazepam following valproic acid. CT image obtained after cesarean section depicted intracerebral hemorrhage in pons and in the left superior cerebellar peduncle (Figure 2a). MR investigation confirmed hemorrhagic focuses without vascular malformation as well as small ischemic focuses (Figure 2b). At 23 days postpartum, the mother and her baby were discharged from the hospital. Patient has been requested directly to rehabilitation center, where she stayed there 22 days. One year later she is in good condition fully independent; in modified Rankin scale (mRs) 1 point.

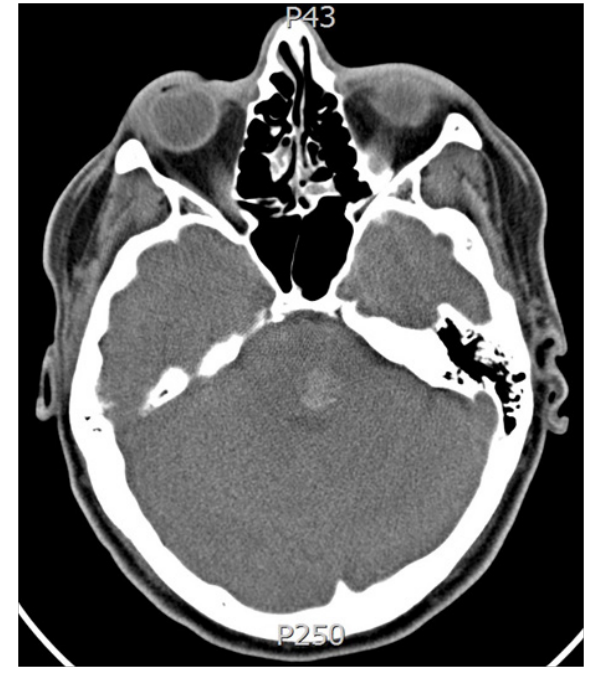

Figure 2a CT image obtained after cesarean section depicted intracerebral hemorrhage in pons.

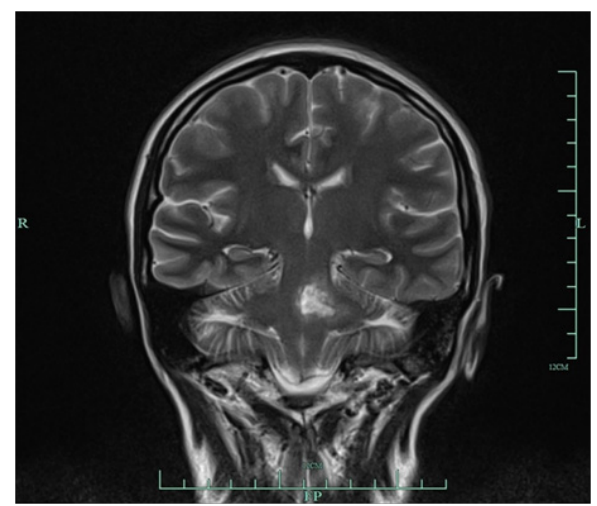

Figure 2b MR investigation confirmed hemorrhagic focuses.

\section{Discussion}

Eclampsia and stroke during pregnancy are major causes of maternal and neonatal death in many countries. ${ }^{1,5}$ This is dangerous disease where hypertension, tonic-clonic seizures as well as neurological deficit (usually hemiparesis) are classic symptoms. ${ }^{7}$ These symptoms require immediate hypotensive treatment and 
intravenous infusion of magnesium sulfate..$^{5,7,10}$ Occasionally in patients with eclampsia HELLP syndrome developing, which can be fatal to both mother and unborn baby. ${ }^{7,12}$ Prompt treatment and delivery of the baby are generally required for the best outcome. ${ }^{10}$ In eclampsia, especially when coexists HELLP syndrome, it can lead to the stroke, which would further deteriorate and so serious situation. Mostly there is intracerebral haemorrhage; however often in the same patient are both hemorrhages and ischaemic foci what was observed in case one. Intracerebral haemorrhage is caused by hypertension and haemorrhagic diathesis due to disseminated intravascular coagulation (DIC) while ischaemic lesions arise due to create parietal blood clots in shrinking vessels. ${ }^{12}$ At the time of stroke is appeared in a patient with eclampsia it seems that this condition can be considered as critical.

In such situation a close collaboration with many specialists is indispensable what underlines many authors., ${ }^{2,10,13}$ The treatment process in our patients was attended by: neurologist, gynecologist, anesthesiologist and hematologist. In both patients after treatment blood parameters normalised. Neurosurgeon consulted also these patients but they did not require surgical treatment. In both cases, the action was fast, multidirectional, and cesarean section was performed extremely well in a very short period of time after arrival at the hospital. We would like emphasise that pregnant patients with eclampsia and HELLP syndrome should be given as soon as possible, in such center, where it is possible to create comprehensive treatment including neurosurgical. Presented patients, treated in a specialist hospital had such care provided.

\section{Acknowledgments}

None.

\section{Conflicts of interest}

The authors declare there is no conflict of interests.

\section{References}

1. Sidiriv EV, Feng W, Caplan LR. Stroke in pregnant and postpartum women. Expert Rev Cardiovasc Ther. 2011;9(9):1235-1247.
2. Prabhu TR. Cerebrovascular complications in pregnancy and puerperium. J Obstet Gynaecol India. 2013;63(2):108-111.

3. Kevat D, Mackillop L. Neurological diseases in pregnancy. $J R$ Coll Physicions Edinb. 2013;43(1):49-58.

4. Feske SK, Singhal AB. Cerebrovascular disorders complicating pregnancy. Continuum (MineapMinn). 2014;20(1 Neurology of Pregnancy):80-9.

5. Kullberg G, Lindeberg S, Hanson U, Eclampsia in Sweden. Hypertens Pregnancy. 2002;21(1):13-21.

6. Kobayashi $\mathrm{T}$, Tokunaga $\mathrm{N}$, Isoda $\mathrm{H}$, et al. Vasospasms are characteristic in cases with eclampsia/preeclampsia and HELLP Syndrome: proposal of an angiospastic syndrome of pregnancy. Semin Thromb Hemost. 2001;27(2):131-136.

7. Bezircioglu I, Baloglu A, Cetinkaya B, et al. Do clinical and laboratory parameters effect maternal and fetal outcomes in pregnancies complicated with hemolysis, elevated liver enzymes, and low platelet count syndrome? J Turkish-German Gynecol Assoc. 2012;13(1):1-7.

8. Hund M, Allegranza D, Schoedl M, et al. Multicenter prospective clinical study to evaluate the prediction of short-term outcome in pregnant women with suspected preeclampsia (Prognosis): study protocol. $B M C$ Pregnancy Childbirth. 2014;14:324.

9. Takahashi JC, Iihara K, Ishii A, et al. Pregnancy-associated intracranial hemorrhage: results of a survey of neurosurgical institutes across Japan. J Stroke Cerebrovasc Dis. 2014;23(2):e65-e71.

10. Ohno Y, Kawai M, Morikawa S, et al. Management of eclampsia and stroke during pregnancy. Neurol Med Chir (Tokyo). 2013;53(8):513-519.

11. Crovetto F, Somigliana E, Peguero A, et al. Stroke during pregnancy and pre-eclampsia. Curr Opin Obstet Gynecol. 2013;25(6):425-432.

12. Foo L, Bewley S, Rudd A. Maternal death from stroke: a thirty year national retrospective review. Eur J Obstet Gynecol Reprod Biol. 2013;171(2):266-270.

13. Edlow JA, Caplan LR, O’Brien K, et al. Diagnosis of acute neurological emergencies in pregnant and post-partum women. Lancet Neurol. 2013;12(2):175-185. 